\title{
Magnetic microemulsions based on magnetic ionic liquids $\dagger$
}

\author{
Andreas Klee, ${ }^{* a}$ Sylvain Prevost, ${ }^{a b}$ Werner Kunz, ${ }^{c}$ Ralf Schweins, ${ }^{d}$ Klaus Kiefer ${ }^{b}$ \\ and Michael Gradzielski ${ }^{* a}$
}

Received 10th July 2012, Accepted 13th September 2012
DOI: 10.1039/c2cp43048g

Microemulsions with magnetic properties were formed by employing a magnetic room temperature ionic liquid (MRTIL) as polar phase, cyclohexane as oil, and an appropriate mixture of ionic surfactant and decanol as a cosurfactant. By means of small-angle neutron scattering (SANS) and electric conductivity the microemulsion structure could be confirmed, where the classical structural sequence of oil-continuous-bicontinuous-polar phase continuous is observed with increasing ratio [polar phase]/[oil]. Accordingly a maximum of the structural size is observed at about equal volumes of oil and MRTIL contained. Therefore this system is structurally the same as normal microemulsions but with the magnetic properties added to it by the incorporation into the systems formulation.

\section{Introduction}

Microemulsions are thermodynamically stable systems typically formed from oils and water by the presence of a surfactant. Normally they are low viscous and can be of the water-in-oil $(\mathrm{W} / \mathrm{O})$, oil-in-water $(\mathrm{O} / \mathrm{W})$, or bicontinuous structural type. ${ }^{1}$ Room temperature ionic liquids (RTILs) have been intensely investigated during recent years and also microemulsions have been formulated with them. This has mostly been done for RTIL replacing water ${ }^{2-11}$ but it is also possible that the RTIL functions as the hydrophobic component of the microemulsion. ${ }^{12,13}$ However, in any case the range of applicable surfactant is much more restricted than in the case of water. A rather novel subclass of RTILs are the so-called magnetic RTILs (MRTILs) $\ddagger$, which were first described in $2004^{14}$ and so far only few publications regarding their properties exist. ${ }^{15-27}$ In particular, none in which they have been employed as a main component in self-aggregating systems, despite the fact that, due to their magnetic properties, they are very interesting and promising compounds. In that context it might also be mentioned that "magnetic surfactants" have received high attention very recently. ${ }^{28}$

\footnotetext{
${ }^{a}$ Stranski-Laboratorium für Physikalische und Theoretische Chemie, Institut für Chemie, Straße des 17. Juni 124, Sekr. TC7, Technische Universität Berlin, 10623 Berlin, Germany.

E-mail: michael.gradzielski@tu-berlin.de; Fax: + 4930314 26602,

Tel: +493031424934

${ }^{b}$ Helmholtz-Zentrum Berlin für Materialien und Energie,

Hahn-Meitner-Platz 1, 14109 Berlin, Germany

${ }^{c}$ Institute of Physical and Theoretical Chemistry,

University of Regensburg, 93040 Regensburg, Germany

${ }^{d}$ Institute Laue Langevin, F-38042 Grenoble, France

$\dagger$ Electronic supplementary information (ESI) available: Experimental section, viscosity data, detailed description of Teubner-Strey and core-shell fits. See DOI: $10.1039 / \mathrm{c} 2 \mathrm{cp} 43048 \mathrm{~g}$

$\ddagger$ It should be noted that they are paramagnetic compounds.
}

Accordingly, self-assembly in MRTIL is an interesting question and in this work we describe the formulation of a magnetic microemulsion based on a MRTIL, which to the best of our knowledge, is the first time that such a system is described. Other microemulsions with magnetic properties are all based on microemulsions containing magnetic inorganic nanoparticles, which is a very different type of formulation. ${ }^{29-31}$ Therefore this magnetic microemulsion based on a MRTIL is a novel type of self-assembled system which is formed by employing an appropriate combination of surfactant and cosurfactant. In the following we describe the phase behavior observed, the magnetic properties and a thorough structural characterisation of this magnetically responsive colloidal system.

\section{Experimental section}

bmim $\left[\mathrm{FeCl}_{4}\right]$ was synthesized as described in the literature. ${ }^{14}$ Briefly bmimCl (24.8 g, $141.9 \mathrm{mmol}$, Aldrich, $\geq 95 \%)$ was heated to $90{ }^{\circ} \mathrm{C}$ in a glass flask under stirring. After adding an equal amount of $\mathrm{FeCl}_{3} \cdot 6 \mathrm{H}_{2} \mathrm{O}(38.4 \mathrm{~g}, 141.9 \mathrm{mmol}$, Sigma Aldrich) the mixture was cooled to room temperature and stirred for $1 \mathrm{~h}$. Removal of the water phase leads to $41.7 \mathrm{~g}$ (124.0 mmol, $87.4 \%$ ) product. $\mathrm{C}_{16} \mathrm{mimCl}$ was synthesized as described in ref. 32 .

The pseudo ternary phase diagram was recorded at a temperature of $24 \pm 0.5^{\circ} \mathrm{C}$. Mixtures of different ratios between cyclohexane and surfactant-co-surfactant were titrated with bmim $\left[\mathrm{FeCl}_{4}\right]$. In all cases the molar ratio of $\mathrm{C}_{16} \mathrm{mimCl} /$ decanol was $1: 2$.

Conductivity titration was done at a temperature of $24{ }^{\circ} \mathrm{C}$ with a home-build Pt-electrode connected to a Methrom 712 conductometer at $2.4 \mathrm{kHz}$, starting with an oil rich microemulsion sample by stepwise addition of an oil free sample with a syringe. 
Small angle neutron scattering (SANS) experiments were performed at ILL, Grenoble (D11). For better contrast the samples were prepared with D12-cyclohexane and for the measurements placed in cuvettes (Quarz, Hellma) of $1 \mathrm{~mm}$ thickness and studied with an incoming beam of $6 \AA$ wavelength and a collimation length of $8 \mathrm{~m}$. Scattered neutrons were recorded using a 2D-detector for sample-to-detector distances of $8 \mathrm{~m}$ and $1.2 \mathrm{~m}$ resulting in an observed $q$-range of $0.09 \mathrm{~nm}^{-1} \leq q \leq 5.12 \mathrm{~nm}^{-1}$. Incoherent scattering of water was used to correct the detector efficiency and to bring the data to an absolute scale. Data reduction was done with $\mathrm{LAMP}^{33}$ and fitting with SASfit. ${ }^{34}$

Magnetic susceptibility measurements were done at $300 \mathrm{~K}$ with a MPMS (Quantum Design, located at the Laboratory for Magnetic Measurements, HZB). Samples were placed in a home-made vacuum-sealed sample chamber and scanned in a range from -5 to 5 Tesla. The empty cell signal was subtracted from each measurement. All samples showed a linear dependency of magnetization vs. magnetic field in the measured range. Examples are shown in Fig. S1 (ESI $\dagger$ ).

Viscosity measurements (Fig. S2, ESI $\dagger$ ) were done at $25^{\circ} \mathrm{C}$ using a micro-Ostwalt viscosimeter (Ic or IIc, SI Analytics, Mainz). The obtained kinematic viscosity was multiplied with the density of the same sample (Density meter DMA 4500, Anton Paar) to calculate the dynamic viscosity.

\section{Results and discussion}

For our experiments we employed 1-butyl-3-methylimidazolium tetrachloroferrate (bmim $\left.\left[\mathrm{FeCl}_{4}\right]\right)$ as MRTIL and cyclohexane as a simple oil, which has been shown before to have rather solubilisation capacities in microemulsion systems, ${ }^{35,36}$ which also applies to ones in RTIL. ${ }^{37}$ As surfactants different types (such as SDS, AOT, and various $\mathrm{C}_{i} \mathrm{E}_{j}$ ) were initially tested but by far the best performance was observed for 1-hexadecyl-3methylimidazolium chloride $\left(\mathrm{C}_{16} \mathrm{mimCl}\right)$ which is an ionic liquid itself but due to the long alkyl chain also a cationic surfactant. ${ }^{32}$ With the $\mathrm{C}_{16} \mathrm{mimCl}$ the formulation of a microemulsion with the aim of using as little surfactant as possible was achieved. However, by itself it is not able to form extended microemulsion areas with $\operatorname{bmim}\left[\mathrm{FeCl}_{4}\right]$ and cyclohexane. Therefore a cosurfactant, in our case decanol (which is insoluble in $\operatorname{bmim}\left[\mathrm{FeCl}_{4}\right]$ ), had to be added to the formulation as is a typical recipe for microemulsions. ${ }^{1,32}$

\subsection{Phase behavior}

The phase behavior of the quaternary system $\operatorname{bmim}\left[\mathrm{FeCl}_{4}\right]_{-}^{-}$ $\mathrm{C}_{16}$ mimCl-decanol-cyclohexane was studied in thorough detail and Fig. 1 shows the pseudo ternary phase diagram of this system for a constant $\mathrm{C}_{16} \mathrm{mimCl} / \mathrm{decanol}$ mole ratio of $1: 2$. This particular ratio gave a maximum for the mixing efficiency of cyclohexane and $\operatorname{bmim}\left[\mathrm{FeCl}_{4}\right]$ and was therefore chosen. In the following we consider $\mathrm{C}_{16} \mathrm{mimCl}$ and decanol as a combined effective amphiphile. Compared to the examples of IL containing microemulsions in the literature, ${ }^{4,5,10}$ which require $40 \mathrm{wt} \%$ more of amphiphile for the formation of a balanced microemulsion, our formulation requires much less stabilizing amphiphile and microemulsions for surfactantcosurfactant amounts less than $20 \mathrm{wt} \%$ are observed. The phase diagram shows a very large and optically clear single

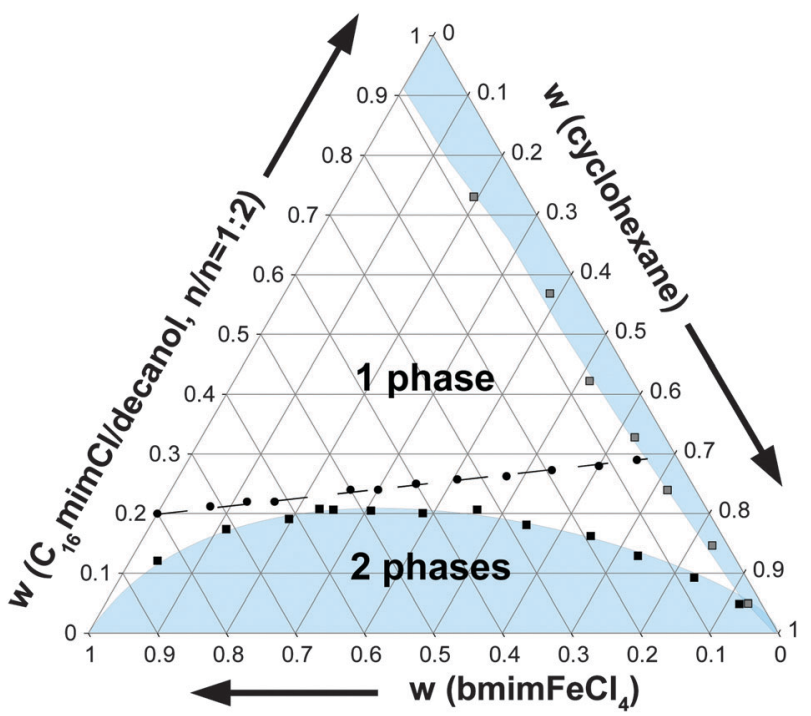

Fig. 1 Pseudo ternary phase diagram (by weight) for mixtures of MRTIL (bmim[ $\left[\mathrm{FeCl}_{4}\right]$ ) and cyclohexane formulated with $\mathrm{C}_{16} \mathrm{mimCl}$ and decanol (molar ratio $1: 2$ ) as surfactant and co-surfactant, respectively. The filled squares mark the detected phase transitions and the filled circles indicate the SANS samples. The dashed line gives the experimental path for conductivity titration and magnetic measurements.

phase region. Viscosities (see ESI $\dagger$ ) are low and in the range obtained for assuming a linear relation for the MRTILcyclohexane mixture viscosities, i.e., they are determined simply by the liquid components of the microemulsion. The low viscosity already hints at the presence of a microemulsion in this range and against formation of a liquid crystalline phase, which is further confirmed by the fact of optical isotropy. Two multi phase regions are observed. One at low MRTIL content due to a limited solubility of the surfactant in the oil resulting in a coexistence of microemulsion and solid $\mathrm{C}_{16} \mathrm{mimCl}$. However, this region becomes monophasic at higher temperatures. The multiphase region at low amphiphile concentrations consists of coexisting oil- and MRTIL-rich phases, a behavior which is well known in classical water containing microemulsions as emulsification failure. ${ }^{38}$ Here it might be noted that the phase behavior and the microemulsions themselves are not sensitive to water, which means they are stable under moist conditions.

\subsection{Magnetic behavior}

Of course, the most interesting aspect of this microemulsion is its magnetic properties, which are depicted in Fig. 2, which shows how the meniscus of the magnetic microemulsion is moved by the presence of the magnetic field, i.e., it is magnetically responsive and this applies to the whole microemulsion range that contains at least a few percent of MRTIL. A more quantitative insight has been gained by measurements of the magnetic susceptibility, which was done along the dashed line given in Fig. 1 covering the full range from the pure oil to pure MRTIL as solvent. All samples show a paramagnetic behavior indicated by a linear field dependency of the magnetization (see Fig. S1, ESI $\dagger$ ). The magnetic susceptibility (inset in Fig. 3) increases in an almost perfectly linear fashion with the increase in weight content of the MRTIL, which means that the magnetic 


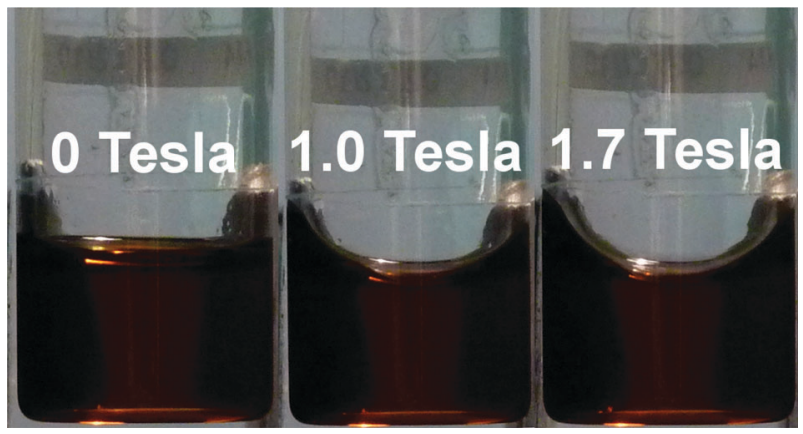

Fig. 2 Response of the MRTIL containing microemulsion to the field gradient of an electromagnet. The sample shown consists of $31.2 \mathrm{wt} \%$ D12-cyclohexane, $46.1 \mathrm{wt} \% \operatorname{bmim}\left[\mathrm{FeCl}_{4}\right], 11.8 \mathrm{wt} \% \mathrm{C}_{16} \mathrm{mimCl}$ and $10.9 \mathrm{wt} \%$ decanol. The magnetic field is oriented parallel to the liquid surface.

properties of the microemulsion are not affected by its mesoscopic structure. Extrapolation to pure bmim $\left[\mathrm{FeCl}_{4}\right]$ gives a magnetic susceptibility of $3.6 \times 10^{-4}$ (SI units). For comparison the initial susceptibility of ferrofluids can be found to be of the order of $1 .{ }^{39}$

\subsection{Mesoscopic structure}

A first insight into this mesoscopic microemulsion structure can be deduced from the conductivity titration shown in Fig. 3. The position in the phase diagram is given by the MRTIL volume ratio $x_{\text {MRTIL }}$ relative to the volume of cyclohexane $\left(V_{\text {oil }}\right)$ and MRTIL ( $\left.V_{\text {MRTIL }}\right)$, defined as:

$$
x_{\text {MRTIL }}=\frac{V_{\text {MRTIL }}}{V_{\text {MRTIL }}+V_{\text {oil }}}
$$

With increasing MRTIL content the conductivity increases by one order of magnitude, which is due to the formation of a structure continuous in MRTIL. In water and oil containing microemulsions as well as in systems with an IL substituting the water such a percolation behavior of the conductivity is well known and can be explained by structural transitions

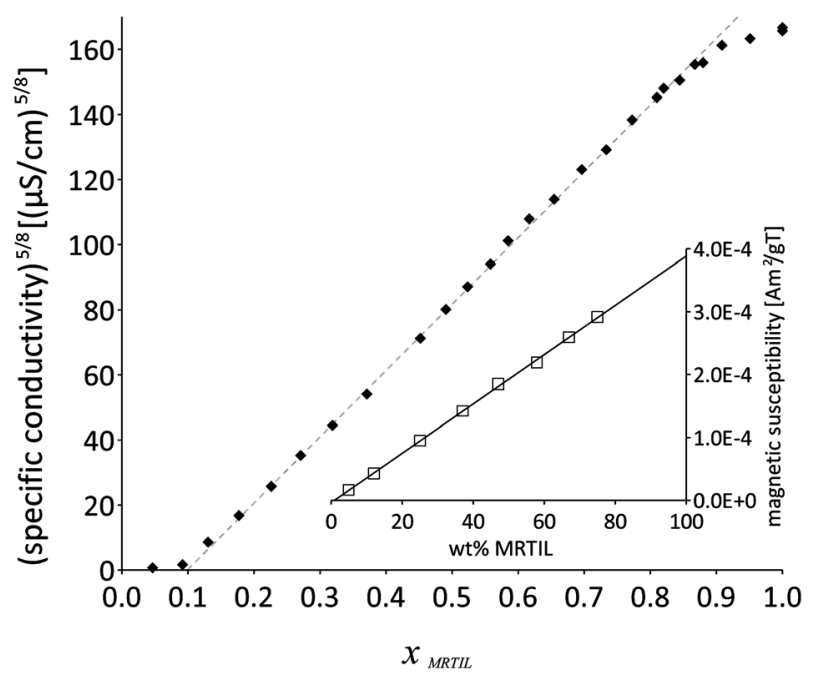

Fig. 3 Specific electric conductivity as a function of the MRTIL volume ratio (eqn (1)) (filled diamonds) and magnetic susceptibility as a function of wt $\%$ MRTIL (inset, open squares). Measurements were done along the experimental path shown in Fig. 1. between a droplet and a bicontinuous structure. ${ }^{5}$ The behavior of our MRTIL system is analogous and shows a percolation point of conductivity around $x_{\mathrm{MRTIL}} \approx 0.1$, determined by plotting the specific conductivity with an exponent of $5 / 8$ versus $x_{\text {MRTIL }}$ (Fig. 3). ${ }^{32,40}$

In order to gain a more detailed structural insight we performed small-angle neutron scattering (SANS). Experiments were performed with D12-cyclohexane in order to have good contrast between oil and MRTIL (the macroscopic phase behavior was not affected by this isotopic substitution). The obtained scattering curves (Fig. 4) show weak correlation peaks for small amounts of MRTIL contained and continuously decreasing intensity curves for higher MRTIL content. The high scattering intensity, especially for about equal contents of oil and MRTIL, and the moving of the scattering curves to lower $q$ prove the existence of relatively large, microphase seperated, domains, as typically observed for microemulsions. Similar scattering patterns have been observed for conventional microemulsions ${ }^{41,42}$ and also for ones containing RTIL. ${ }^{2-4,32}$ It is interesting to note that the degree of ordering is substantially
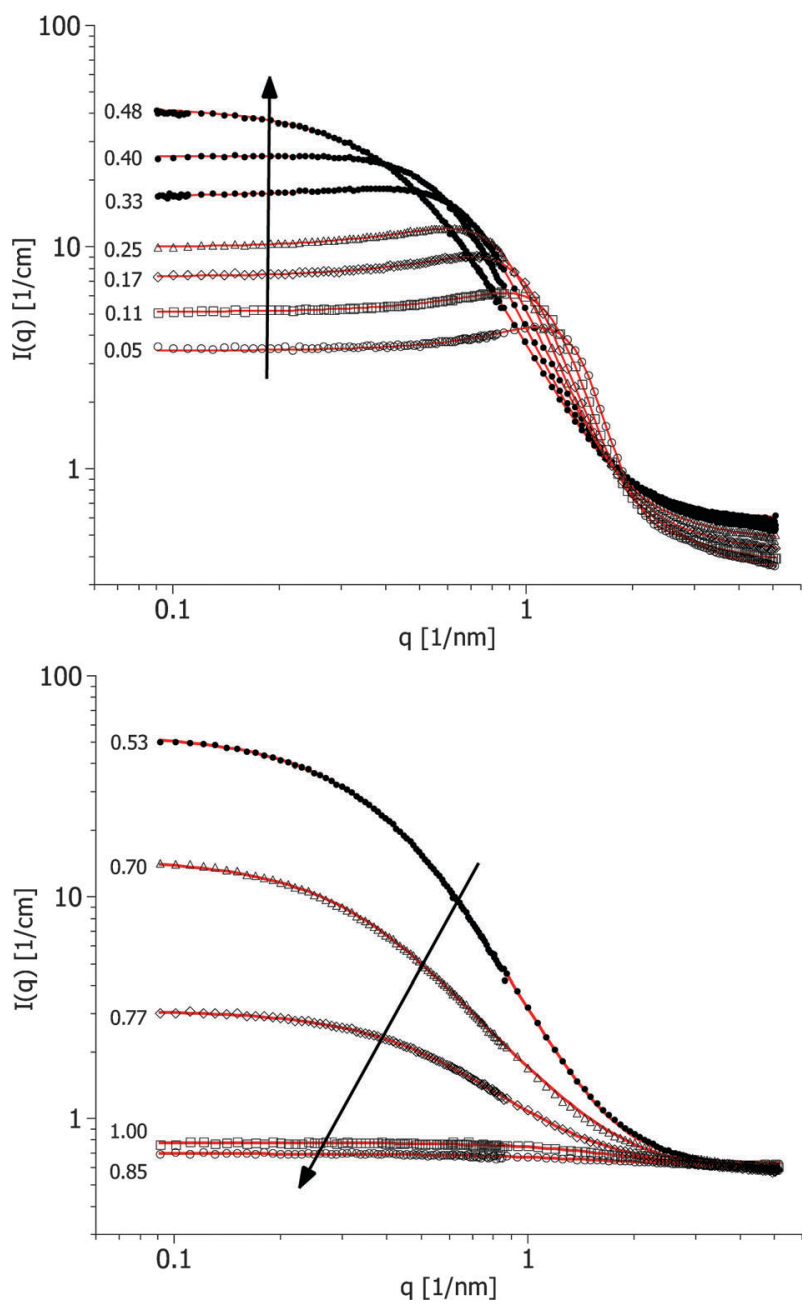

Fig. 4 SANS curves with D12-cyclohexane as oil phase. The samples are along the experimental path shown in Fig. 1. Labels are values for $x_{\text {MRTIL }}$ (eqn (1)), arrows indicating growing $x_{\text {MRTIL }}$. Symbols are measured scattering intensities, lines are fits performed with the TS-model (filled circles) and the sphere model (open symbols). 
lower for the MRTIL rich region as evidenced by the absence of a correlation peak. This is different from water-based microemulsions and can be explained by the much reduced electrostatic repulsion as the RTIL screens effectively the charge of the microemulsion droplets formed.

In order to deduce quantitative structural information from the SANS curves we applied two models to describe the data: first the phenomenological Teubner-Strey (TS) model, ${ }^{43}$ in which the scattering intensity is given by eqn (2a) and is basically determined by the quasiperiodic repeat distance $D_{\mathrm{s}}$ (eqn (2b)) and the correlation length $\xi$ (eqn (2c)) of the structural units, where $\left\langle\eta^{2}\right\rangle$ accounts for the contrast and volume fractions $\Phi$ of the oil and MRTIL phase (eqn (2d)).

$$
\begin{gathered}
I(q)=\frac{8 \pi c_{2}\left\langle\eta^{2}\right\rangle / \xi}{a_{2}+c_{1} q^{2}+c_{2} q^{4}} \\
D_{\mathrm{s}}=2 \pi\left[\frac{1}{2} \sqrt{\frac{a_{2}}{c_{2}}}-\frac{1}{4} \frac{c_{1}}{c_{2}}\right]^{-1 / 2} \\
\xi=2 \pi\left[\frac{1}{2} \sqrt{\frac{a_{2}}{c_{2}}}+\frac{1}{4} \frac{c_{1}}{c_{2}}\right]^{-1 / 2} \\
\left\langle\eta^{2}\right\rangle=\Phi_{\mathrm{IL}} \Phi_{\mathrm{oil}}(\Delta \rho)^{2}
\end{gathered}
$$

As second model we employed a core-shell model of spherical droplets, described by a particle form factor $P$ and a structure factor $S$ (in our case for hard spheres ${ }^{44}$ ) (eqn (3)), which are determined by core radius $R_{\mathrm{c}}$, shell thickness $\delta R$, polydispersity index $p$, and hard sphere radius $R_{\mathrm{HS}}$ that accounts for the repulsive interparticle interactions (for details see ESI $\dagger$ ).

$$
I(q)=N P\left(q, R_{\mathrm{c}}, \delta R, p\right) S\left(q, R_{\mathrm{HS}}\right)
$$

Both models allow for a reasonable description of the SANS data but the quality of the agreement of the respective model depends on the location of the sample in the phase diagram (see ESI $\dagger$ ).

The TS model works best in the range of intermediate compositions $\left(x_{\text {MRTIL }}=0.3-0.65\right)$, where a bicontinuous structure is to be expected. For locations in the phase diagram rich in MRTIL or oil the droplet picture of the core-shell model is clearly superior. For the droplet regime one finds core radii that increase with increasing content of solubilisate, being somewhat larger on the oil-rich side. This can be explained by the fact that here the head group of the surfactant has to be counted into the core (see Fig. 5). This explains also why here the shell thickness is correspondingly smaller and it should also be kept in mind that this thickness is not the thickness of the amphiphilic monolayer, as it corresponds only to a part of the length of the ionic surfactant. In general, of course, the core-shell model is superior for large $q$, as it captures better the local structure than the TS model that assumes a sharp interface. The parameters obtained are summarized in Table 1 and nicely show the increase in structural size upon approaching a balanced microemulsion.

In comparison, the TS analysis yields values of 1.7 to $2.3 \mathrm{~nm}$ for the correlation length $\xi$. In contrast the repeat distance $D_{\mathrm{s}}$ increases from 5.4 to $60 \mathrm{~nm}$ (the $60 \mathrm{~nm}$ is a lower realistic estimate as this corresponds to the maximum of the experimental

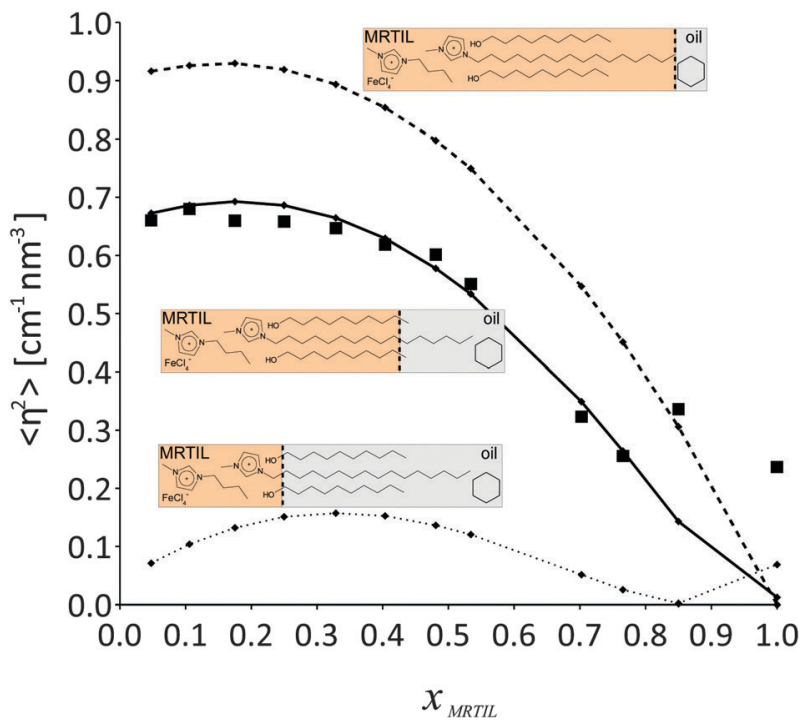

Fig. $5\left\langle\eta^{2}\right\rangle$ as deduced from the TS fits (filled squares) compared to values calculated with eqn (2d) from the real sample compositions for three possible devisions of the interface: The whole interface is counted into the IL phase (dashed line), all hydrocarbon chains of the surfactant-cosurfactant belong to the oil phase (dotted line), and partial partitioning of the hydrocarbon chains (full line).

observation window) (Table 1) with increasing $x_{\text {MRTIL }}$ until a maximum is reached for the balanced microemulsion $\left(x_{\text {MRTIL }}=0.5\right)$. From this relatively low value of $\xi$ one can conclude that the bending rigidity of the amphiphilic interface in the bicontinuous phase must be rather low as $\xi$ is approaching the effective length of the surfactant. ${ }^{45}$ This can be quantified further by calculating the renormalized bending rigidity $v i a^{46,47}$

$$
\frac{\kappa}{k_{\mathrm{B}} T}=\frac{10 \sqrt{3} \pi}{64} \frac{\xi}{D_{\mathrm{s}}}
$$

which shows that $\kappa$ goes down from $0.26 k_{\mathrm{B}} T$ for $x_{\mathrm{MRTIL}}=$ 0.17 to $0.058 k_{\mathrm{B}} T$ for $x_{\mathrm{MRTIL}}=0.48$ (balanced microemulsion).

Table 1 Parameters for the TS fits, correlation length $\xi$, repeat distance $D_{\mathrm{s}}$ and calculated amphiphilicity factor $f_{\mathrm{a}}=c_{1} / \sqrt{4 a_{2} c_{2}}$. In addition, core radius $R_{\mathrm{c}}$, shell thickness $\delta R$ and hard-sphere radius $R_{\mathrm{HS}}$ of the core-shell model are given. For samples with $x_{\text {MRTIL }}=0.85$ and 1.00 no structure factor $S(q)$ was necessary. Parameters for fits not shown in Fig. 4 are in italics

\begin{tabular}{llllllll}
\hline & \multicolumn{2}{l}{ TS model } & & \multicolumn{2}{c}{ Core-shell model } \\
\cline { 2 - 3 } \cline { 6 - 7 }$x_{\text {MRTIL }}$ & $\xi / \mathrm{nm}$ & $D_{\mathrm{s}} / \mathrm{nm}$ & $f_{\mathrm{a}}$ & & $R_{\mathrm{c}} / \mathrm{nm}$ & $\delta R / \mathrm{nm}$ & $R_{\mathrm{HS}} / \mathrm{nm}$ \\
\hline 0.05 & 2.05 & 5.44 & -0.70 & 1.13 & 0.58 & 2.11 \\
0.11 & 1.90 & 6.53 & -0.54 & 1.21 & 0.57 & 2.46 \\
0.17 & 2.29 & 7.48 & -0.58 & 1.32 & 0.58 & 2.87 \\
0.25 & 2.28 & 8.59 & -0.47 & 1.50 & 0.45 & 3.37 \\
0.33 & 2.29 & 10.76 & -0.28 & 1.57 & 0.43 & 3.98 \\
0.40 & 2.30 & 13.39 & -0.07 & 2.80 & 0.95 & 4.92 \\
0.48 & 1.86 & 27.36 & 0.69 & 2.53 & 0.97 & 6.16 \\
0.53 & 1.95 & 60.00 & 0.92 & 2.41 & 1.07 & 6.01 \\
0.70 & 1.74 & 60.00 & 0.94 & 2.38 & 2.18 & 1.03 \\
0.77 & 1.14 & 60.00 & 0.97 & 1.49 & 1.36 & 0.94 \\
0.85 & 0.36 & 6.00 & 0.75 & 0.44 & 1.03 & - \\
1.00 & 0.65 & 5.64 & 0.32 & 0.00 & $0.89^{a}$ & -
\end{tabular}

${ }^{a}$ This value was described by a lognormal distribution and is calculated from the 3 rd moment similar to $R_{\mathrm{c}}=\sqrt[3]{\left\langle R^{3}\right\rangle}$ (for details see ESI). 
This very low value for $\kappa$ explains why we do not observe a structural correlation peak in the SANS curves of the balanced microemulsion as simply the amphiphilic interface is very soft. This difference to conventional microemulsions can first be attributed to having a very different solvent than water, where the RTIL is much more efficient in reducing long range electrostatic interactions. This is corroborated further by the large increase of the amphiphilicity factor $f_{\mathrm{a}}$ (see Table 1) which indicates that structural ordering of the microemulsion becomes less and less pronounced and for the balanced microemulsion even approaches values that are not so far away from the disorder line, where an unstructured liquid would be formed. ${ }^{48,49}$ Nonetheless in our system large domain structures are present in these solutions as evidenced by the high scattering intensity. In general, this simply means that the surfactant employed here is not a strong amphiphile for the cyclohexane-MRTIL mixture, which is in agreement with the fact that rather large amounts of it are required for forming a microemulsion, as is typically observed for microemulsions with RTIL. ${ }^{4,5,10}$

However, the increase of the structural size and the passing from a MRTIL-in-oil (MRTIL/O) over a bicontinuous to an oil-in-MRTIL (O/MRTIL) structure occurs in our MRTIL microemulsion in a similar way as in conventional microemulsions $^{38}$ (but with a more pronounced increase for high MRTIL content). It must also be noted that the TS model is very sensitive to the detailed structural features that determine the contrast conditions of the structure (eqn (2d)). In Fig. 5 we compare the experimental value of $\left\langle\eta^{2}\right\rangle$ with the ones calculated such that all of the alkyl chains belongs either to the oil phase or the MRTIL phase. Both assumptions show strong deviations from the experimental data and only for a dividing surface placed in the middle, which corresponds exactly to the length of the decanol molecule (see Fig. 5), the intensity is correctly accounted for. This means that our quantitative analysis of the SANS curves allows us to deduce a rather refined picture about the mesoscopic structure, as described by $\left\langle\eta^{2}\right\rangle$ and $D_{\mathrm{s}}$, as well as about the precise local seperation between the hydrophilic and hydrophobic domains.

\section{Conclusion}

In summary, our experiments demonstrate that with the MRTIL bmim[ $\mathrm{FeCl}_{4}$ ] we are able to form microemulsions that are similar to classical oil-water microemulsions with respect to their phase behavior and structure, but show weaker amphiphilicity, softer amphiphilic films and accordingly less structured microemulsions, particularly in the range of balanced microemulsions. Nonetheless large size domains are present as evidenced by the high SANS intensity observed. The most interesting property is that, due to the MRTIL, this is a low viscous "magnetic microemulsion" over the whole range of composition, which can be moved and manipulated by a magnetic field. By proper choice of the surfactant-cosurfactant mixture the amount of amphiphile required for the formation of a large single phase microemulsion region can be kept relatively small, thereby leading to these correspondingly large microemulsion structures. In this region the typical phase inversion (O/MRTIL)-bicontinuous (MRTIL/O) takes place as one changes the ratio between ionic liquid and oil. Such a magnetic microemulsion is a novel type of self-assembled system which due to its magnetic properties may open interesting paths for formulating functional and responsive systems. This even more so as its formulation can be achieved in a very simple fashion. Its properties are very promising as in our system we combine the low viscosity and structural versatility of a microemulsion with magnetic properties without having to resort to incorporating magnetic nanoparticles, which would alter the whole structuring and behavior of the given systems and render it much more complex to handle. Accordingly we expect magnetic microemulsions to become a class of colloidal systems which will have a large impact on future research activities and applications.

\section{Acknowledgements}

The ILL is thanked for allocation of SANS beam time and the Helmholz Zentrum Berlin for performing SQUID measurements. Sebastian Gerischer and Robert Wahle are thanked for their help with the construction of the SQUID liquid cell.

\section{References}

1 P. Kumar, Handbook of microemulsion science and technology, Dekker, 1999, p. 849

2 O. Zech, S. Thomaier, A. Kolodziejski, D. Touraud, I. Grillo and W. Kunz, J. Colloid Interface Sci., 2010, 347, 227-232.

3 J. Eastoe, S. Gold, S. E. Rogers, A. Paul, T. Welton, R. K. Heenan and I. Grillo, J. Am. Chem. Soc., 2005, 127, 7302-7303.

4 R. Atkin, S. M. C. Bobillier and G. G. Warr, J. Phys. Chem. B, 2010, 114, 1350-1360.

5 N. Li, S. H. Zhang, L. Q. Zheng, Y. Gao and L. Yu, Langmuir, 2008, 24, 2973-2976.

6 S. K. Mehta and K. Kaur, Anal. Chem. Indian J. Chem., Sect. A, 2010, 49, 662-684.

7 O. Zech and W. Kunz, Soft Matter, 2011, 7, 5507-5513.

8 L. Liu, P. Bauduin, T. Zemb, J. Eastoe and J. Hao, Langmuir, 2009, 25, 2055-2059.

9 O. Rojas and J. Koetz, J. Surf. Sci. Technol., 2010, 26, 173-196.

10 Y. Gao, L. Hilfert, A. Voigt and K. Sundmachert, J. Phys. Chem. B, 2008, 112, 3711-3719.

11 R. Atkin and G. G. Warr, J. Phys. Chem. B, 2007, 111, 9309-9316.

12 D. Seth, A. Chakraborty, P. Setua and N. Sarkar, Langmuir, 2006, 22, 7768-7775.

13 Y. Gao, N. Li, L. Q. Zheng, X. Y. Zhao, S. H. Zhang, B. X. Han, W. G. Hou and G. Z. Li, Green Chem., 2006, 8, 43- 49.

14 S. Hayashi and H. Hamaguchi, Chem. Lett., 2005, 740.

15 R. E. Del Sesto, T. M. McCleskey, A. K. Burrell, G. A. Baker, J. D. Thompson, B. L. Scott, J. S. Wilkes and P. Williams, Chem. Commun., 2008, 447-449.

16 B. Mallick, B. Balke, C. Felser and A. V. Mudring, Angew. Chem., Int. Ed., 2008, 47, 7635-7638.

17 T. Peppel, M. Kockerling, M. Geppert-Rybczynska, R. V. Ralys, J. K. Lehmann, S. P. Verevkin and A. Heintz, Angew. Chem., Int. Ed., 2010, 49, 7116-7119.

18 Y. Yoshida and G. Saito, J. Mater. Chem., 2006, 16, 1254-1262.

19 M. Okuno, H. O. Hamaguchi and S. Hayashi, Appl. Phys. Lett., 2006, 89, 132506.

20 T. Akitsu and Y. Einaga, Inorg. Chem. Commun., 2006, 9, $1108-1110$.

21 S. H. Lee, S. H. Ha, C. Y. You and Y. M. Koo, Korean J. Chem. Eng., 2007, 24, 436-437.

22 M. Li, S. L. De Rooy, D. K. Bwambok, B. El-Zahab, J. F. DiTusa and I. M. Warner, Chem. Commun., 2009, 6922-6924.

23 A. Getsis, B. Balke, C. Felser and A.-V. Mudring, Cryst. Growth Des., 2009, 9, 4429-4437.

24 J. Y. Kim, J. T. Kim, E. A. Song, Y. K. Min and H. Hamaguchi, Macromolecules, 2008, 41, 2886-2889.

25 S. Shang, L. Li, X. Yang and L. Zheng, J. Colloid Interface Sci., 2009, 333, 415-418. 
26 B. M. Krieger, H. Y. Lee, T. J. Emge, J. F. Wishart and E. W. Castner, Jr., Phys. Chem. Chem. Phys., 2010, 12, 8919-8925.

27 I. de Pedro, D. P. Rojas, J. Albo, P. Luis, A. Irabien, J. A. Blanco and J. R. Fernandez, J. Phys.: Condens. Matter, 2010, 22, 296006.

28 P. Brown, A. Bushmelev, C. P. Butts, J. Cheng, J. Eastoe, I. Grillo, R. K. Heenan and A. M. Schmidt, Angew. Chem., Int. Ed., 2012, 51, 2414-2416.

29 J. A. L. Perez, M. A. Lopez Quintela, J. Mira, J. Rivas and S. W. Charles, J. Phys. Chem. B, 1997, 101, 8045-8047.

30 Z. L. Liu, X. Wang, K. L. Yao, G. H. Du, Q. H. Lu, Z. H. Ding, J. Tao, Q. Ning, X. P. Luo, D. Y. Tian and D. Xi, J. Mater. Sci., 2004, 39, 2633-2636.

31 A. H. Lu, E. L. Salabas and F. Schuth, Angew. Chem., Int. Ed., 2007, 46, 1222-1244.

32 O. Zech, S. Thomaier, P. Bauduin, T. Ruck, D. Touraud and W. Kunz, J. Phys. Chem. B, 2009, 113, 465-473.

33 D. Richard, M. Ferrand and G. J. Kearley, J. Neutron Res., 1996, 4, 33-39.

34 J. Kohlbrecher and I. Bressler, http://kur.web.psi.ch/sans1/SANS Soft/sasfit.html.

35 P. Barois, S. Hyde, B. Ninham and T. Dowling, Langmuir, 1990, 6, 1136-1140.

36 J. C. Panitz, M. Gradzielski, H. Hoffmann and A. Wokaun, J. Phys. Chem., 1994, 98, 6812-6817.
37 R. Pramanik, S. Sarkar, C. Ghatak, V. G. Rao, P. Setua and N. Sarkar, J. Phys. Chem. B, 2010, 114, 7579-7586.

38 M. E. Cates, D. Andelman, S. A. Safran and D. Roux, Langmuir, 1988, 4, 802-806.

39 S. Odenbach, J. Phys.: Condens. Matter, 2004, 16, R1135-R1150.

40 B. Lagourette, J. Peyrelasse, C. Boned and M. Clausse, Nature, 1979, 281, 60-62.

41 O. Regev, S. Ezrahi, A. Aserin, N. Garti, E. Wachtel, E. W. Kaler, A. Khan and Y. Talmon, Langmuir, 1996, 12, 668-674.

42 S. H. Chen, S. L. Chang, R. Strey and P. Thiyagarajan, J. Phys.: Condens. Matter, 1991, 3, F91-F107.

43 M. Teubner and R. Strey, J. Chem. Phys., 1987, 87, 3195-3200.

44 N. W. Ashcroft and J. Lekner, Phys. Rev., 1966, 145, 83-90.

45 T. Sottmann, R. Strey and S. H. Chen, J. Chem. Phys., 1997, 106, 6483-6491.

46 G. Gompper, H. Endo, M. Mihailescu, J. Allgaier, M. Monkenbusch, D. Richter, B. Jakobs, T. Sottmann and R. Strey, Europhys. Lett., 2001, 56, 683-689.

47 O. Holderer, M. Klostermann, M. Monkenbusch, R. Schweins, P. Lindner, R. Strey, D. Richter and T. Sottmann, Phys. Chem. Chem. Phys., 2011, 13, 3022-3025.

48 K. V. Schubert, R. Strey, S. R. Kline and E. W. Kaler, J. Chem. Phys., 1994, 101, 5343-5355.

49 M. Gradzielski, D. Langevin, T. Sottmann and R. Strey, J. Chem. Phys., 1996, 104, 3782-3787. 